\title{
BMJ Open Collaborative maternity and newborn dashboard (CoMaND) for the COVID-19 pandemic: a protocol for timely, adaptive monitoring of perinatal outcomes in Melbourne, Australia
}

\author{
Lisa Hui (1) , 1,2,3,4 Melvin B Marzan, ${ }^{1}$ Stephanie Potenza, ${ }^{5}$ Daniel L Rolnik (1) ,6,7 \\ Joanne M Said (D) , ${ }^{1,8}$ Kirsten R Palmer (1) , ,,7 Clare L Whitehead, ${ }^{1,9}$ \\ Penelope M Sheehan, ${ }^{6,10}$ Jolyon Ford, ${ }^{11}$ Natasha Pritchard, ${ }^{1,2}$ Ben W Mol (i) , 6,7 \\ Susan P Walker (iD) ${ }^{1,2}$
}

To cite: Hui L, Marzan MB, Potenza S, et al. Collaborative maternity and newborn dashboard (CoMaND) for the COVID-19 pandemic: a protocol for timely, adaptive monitoring of perinatal outcomes in Melbourne, Australia. BMJ Open 2021;11:e055902. doi:10.1136/ bmjopen-2021-055902

- Prepublication history and additional supplemental material for this paper are available online. To view these files, please visit the journal online (http://dx.doi.org/10.1136/ bmjopen-2021-055902).

Received 17 August 2021 Accepted 09 November 2021

Check for updates

(C) Author(s) (or their employer(s)) 2021. Re-use permitted under CC BY-NC. No commercial re-use. See rights and permissions. Published by BMJ.

For numbered affiliations see end of article.

Correspondence to

Dr Lisa Hui;

lisa.hui@unimelb.edu.au

\section{ABSTRACT}

Background The COVID-19 pandemic has resulted in a range of unprecedented disruptions to maternity care with documented impacts on perinatal outcomes such as stillbirth and preterm birth. Metropolitan Melbourne has endured one of the longest and most stringent lockdowns in globally. This paper presents the protocol for a multicentre study to monitor perinatal outcomes in Melbourne, Australia, during the COVID-19 pandemic. Methods Multicentre observational study analysing monthly deidentified maternal and newborn outcomes from births $\geq 20$ weeks at all 12 public maternity services in Melbourne. Data will be merged centrally to analyse outcomes and create run charts according to established methods for detecting non-random 'signals' in healthcare. Perinatal outcomes will include weekly rates of total births, stillbirths, preterm births, neonatal intensive care admissions, low Apgar scores and fetal growth restriction. Maternal outcomes will include weekly rates of: induced labour, caesarean section, births before arrival to hospital, postpartum haemorrhage, length of stay, general anaesthesia for caesarean birth, influenza and COVID-19 vaccination status, and gestation at first antenatal visit. A prepandemic median for all outcomes will be calculated for the period of January 2018 to March 2020. A significant shift is defined as $\geq 6$ consecutive weeks, all above or below the prepandemic median. Additional statistical analyses such as regression, time series and survival analyses will be performed for an indepth examination of maternal and perinatal outcomes of interests.

Ethics and dissemination Ethics approval for the collaborative maternity and newborn dashboard project has been obtained from the Austin Health (HREC/64722/ Austin-2020) and Mercy Health (ref. 2020-031).

Trial registration number ACTRN12620000878976; Pre-results.

\section{Strengths and limitations of this study}

- This project is the first clinician-led, multicentre perinatal data collection system for metropolitan Melbourne.

- It complements the state government data collection, with the significant benefits of more timely and flexible reporting of outcomes, and granular detail on emerging areas of concern.

- The study relies on primary source coding of exposure and outcomes from each hospital that have not been internally validated during the study period.

- Data from private maternity hospitals, containing approximately $22 \%$ of Melbourne births, are not available.

- This resource will support data-informed hospital pandemic responses through to the end of 2022.

\section{BACKGROUND}

The COVID-19 pandemic has resulted in a range of unprecedented disruptions to the delivery of maternity care globally. International studies have reported significant changes in perinatal outcomes such as stillbirths and preterm birth during the COVID-19 pandemic, raising concerns about the unintended consequences of pandemic restrictions on mothers and newborns. ${ }^{1-4}$ Women in metropolitan Melbourne endured one of the longest and most stringent pandemic lockdowns in the world (stringency index 92.6/100 for approximately 6 weeks), while continuing to give birth to $>4000$ babies per month ${ }^{5}$ (see figure 1 and online supplemental appendix 1).

The majority of prenatal care in Melbourne $(77.5 \%)$ is conducted through public hospital maternity services, via obstetrician 


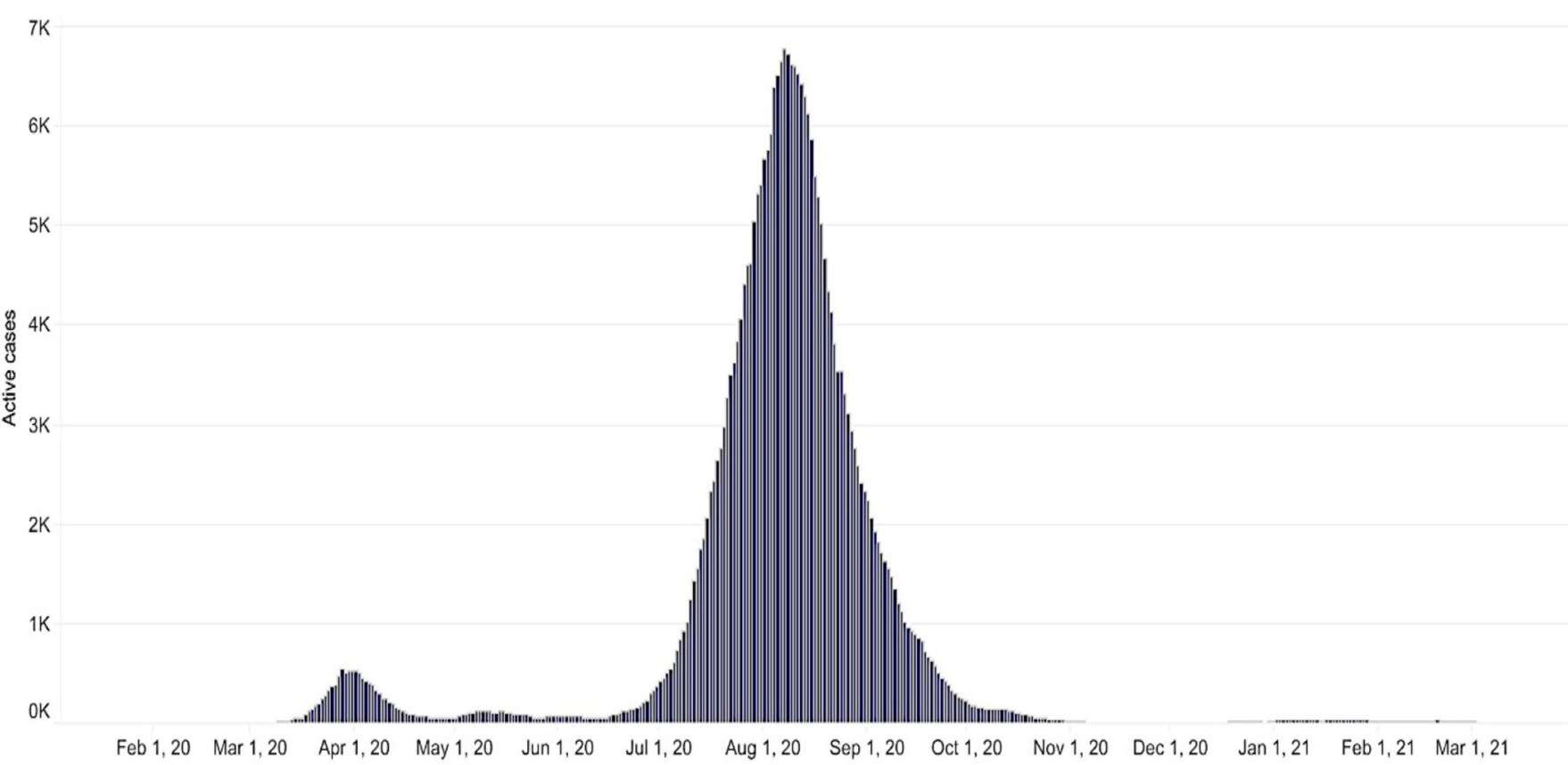

Figure 1 Number of active coronavirus cases by month in Victoria, Australia. Image downloaded from the Victorian Department of Health and Human Services at https://www.dhhs.vic.gov.au/victorian-coronavirus-covid-19-data on 15 March 2021.

or midwifery-lead outpatient clinics or in partnership with hospital accredited 'shared-care' primary care physicians (general practitioners). ${ }^{6}$ The average woman giving birth for the first time has 10-12 antenatal visits and has an average hospital stay of 2-3 days for vaginal birth, or 3-4days after caesarean birth. Australian citizens and permanent residents do not incur any out-of-pocket costs for their inpatient or outpatient episodes of hospital care. Approximately 22.3\% of births occur in private hospitals under the care of a private obstetrician, and $0.2 \%$ occur as planned homebirths under the care of an independent midwife. ${ }^{6}$ These births are not included in this study protocol.

In April 2020, the Royal Australian and New Zealand College of Obstetricians and Gynaecologists released a communique outlining suggested changes to routine pregnancy care to mitigate the anticipated strain on health services and infection risk to patients and healthcare workers. $^{7}$

These measures included:

- Reducing, postponing and/or increasing the interval between antenatal visits.

- Limiting the time of all antenatal visits to less than 15 min.

- Using telehealth consultations as a replacement, or in addition to, routine visits.

- Cancelling face-to-face antenatal classes.

- Limiting visitors (partner only) while in hospital.

- Considering early discharge from hospital.

- Modifications to screening for gestational diabetes.

At the time of implementation, the impact of these widespread changes to antenatal care was unknown. Victorian maternity services collect a comprehensive set of data to fulfil legislative and regulatory requirements. These data are submitted to Safer Care Victoria (SCV) for the biannual Victoria's Mothers, Babies and Childrens Report and the Perinatal Services Performance Indicators (PSPI) report. ${ }^{8}$ However, there is typically a minimum 18 -month lag time between data submission and report publication.

The primary aim of this study is to leverage routinely collected data from all public maternity units in Melbourne to create a timely, flexible monitoring system to monitor perinatal outcomes during the COVID-19 pandemic. Weekly trends in key maternal and newborn outcomes before, during and after the COVID-19 pandemic will be analysed through monthly data uploads from participating health services. The initial study period will include 2 years preceding the WHO declaration of the 'Public Health Emergency of International Concern' in January $2020^{9}$ for baseline prepandemic rates of key performance indicators. As there is uncertainty around the duration of the pandemic, the study will extend to at least 31 December 2022 to account for the length of gestation, lag effects, COVID-19 vaccination roll-out and ongoing alterations to maternity care through 2020 and 2021. The secondary aim of this study is to investigate the effect of lockdowns on outcomes of interest, including preterm birth, stillbirth and birth weight.

\section{METHODS}

\section{Study design}

This multicentre observational study will gather routinely collected data on births in public maternity hospitals in Victoria from 1 January 2018 to 31 December 2022. The first half of the study period (from 1 January 2018 
to commencement of study following ethics and local governance approvals in mid-2020) will be retrospectively collected to provide baseline prepandemic medians rates of all outcomes. Data will then be prospectively collected each calendar month from each of the participating sites, and this will be updated to provide regular reports on maternity and perinatal services.

\section{Participating health services}

The participating health services and contributing hospitals are:

- Mercy Health (Mercy Hospital for Women, Werribee Mercy Hospital).

- The Royal Women's Hospital, The Women's at Sandringham.

- Monash Health (Monash Medical Centre, Casey Hospital, Dandenong Hospital).

- Northern Health (The Northern Hospital).

- Western Health (Joan Kirner Women's and Children's Hospital).

- Eastern Health (Box Hill Hospital, The Angliss Hospital).

- Peninsula Health (Frankston Hospital).

These seven health services (12 hospitals) include four level 6 maternity centres, three-level 5 centres and five-level 4 health services. Level 4 centres provide local care for low-risk mothers and babies including births from 34 weeks' gestation. Levels 5 centres provide care for women with normal to moderate risk pregnancies, including births from 31 weeks' gestation. Level 6 centres provide comprehensive maternity care for women of any risk level, including maternal-fetal medicine services and level 6 newborn care for babies $<31$ weeks' gestation. The detailed definitions of each service capability level are described elsewhere. ${ }^{10}$

\section{Study population}

All births $\geq 20$ weeks with babies with a birth weight $>150$ $\mathrm{g}$ recorded at one of the participating maternity hospitals between 1 January 2018 and 31 December 2022 will be included. Birth $<20$ weeks gestation are excluded as these data are not captured in the current maternity data collection systems. An estimated 44000 women give birth in the participating hospitals each year ${ }^{10}$; the number of births captured during the full study period (2018-2022) will be approximately 220000 . Data from private hospitals will not be collected. Our participating sites will therefore capture approximately $74 \%$ of all hospital births in metropolitan Melbourne, including the highest risk pregnancies requiring referral to level 6 centres (table 1). The age profile of mothers in Victoria is similar to the Australian average, with $38 \%$ of women giving birth being aged 30-34 years old. ${ }^{11}$

\section{Data sources}

There are two sources of data.

Maternity hospital data collection systems

Perinatal outcomes will be collected from the electronic Birthing Outcomes System ${ }^{\mathrm{TM}}$ (BOS, V.6.04, Management Consultants and Technology Services, Caulfield, Victoria, Australia. V.6.04, Management Consultants and Technology Services, Caulfield, Victoria, Australia. https://www.mcats.com.au/) (or equivalent) at each participating hospital. Each BOS site will house identical embedded report queries that will be run monthly with minimal resource requirements (less than 1 hour of work per month to generate output). No individual identifying data (eg, date of birth, maternal date of birth, medical record number) will be collected for this study. Eleven of the 12 participating hospitals use the BOS, ensuring consistent data definitions and immediate pooling of data. The Royal Women's Hospital uses a bespoke database- the RHW data will be cleaned and coded to align

\begin{tabular}{llcc}
\hline \multicolumn{2}{l}{ Table 1} & Metropolitan public hospital births in $2018^{10}$ & \\
\hline Hospital & Service capability level & No of women & No of babies \\
\hline Mercy Hospital for Women & 6 & 5582 & 5693 \\
The Royal Women's Hospital (Melbourne) & 6 & 7472 & 7660 \\
Monash Medical Centre & 6 & 3894 & 4060 \\
The Northern Hospital & 5 & 3649 & 3687 \\
Joan Kirner Women and Children's Hospital & 5 & 5446 & 5524 \\
Box Hill Hospital & 5 & 2596 & 2635 \\
Werribee Mercy Hospital & 4 & 3836 & 3859 \\
The Women's at Sandringham & 4 & 1613 & 1614 \\
Dandenong Hospital & 4 & 2673 & 2673 \\
Casey Hospital & 4 & 2326 & 2326 \\
Angliss Hospital & 4 & 2218 & 2227 \\
Frankston Hospital & 5 & 2869 & 2895 \\
Total births & & 44174 & 44853 \\
\hline
\end{tabular}


with BOS data fields prior to merging with the complete dataset.

A comprehensive set of variables are being collected to enable granular reporting on outcomes, including, but not limited to, those listed below. The complete list of variables is listed in online supplemental appendix 2. As this study has a flexible, adaptive design, additional outcomes can be generated from the list of collected variables as required. Outcomes may be unadjusted or adjusted for terminations of pregnancy and congenital anomalies as appropriate. The key minimum indicators are listed below:

Health system outcomes

- Total births per week.

- Total pregnancies per week.

Maternal outcomes

- First antenatal visit.

- Gestational diabetes.

- Pre-eclampsia (as indication for induction of labour or caesarean birth).

- Multiple pregnancies.

- Maternal body mass index.

- Labour onset.

- Mode of birth.

- Planned setting of birth.

- Maternal influenza vaccination rates.

- COVID-19 vaccination rates.

- Smoking cessation.

- Third and fourth degree perineal tears.

- General anaesthesia for caesarean births.

- Length of stay.

- Postpartum haemorrhage.

Fetal and neonatal outcomes

- Stillbirth rates.

- Preterm births.

- Birth weight $\leq 3 r$ d centile.

- Rate of Apgar scores $<7$ at 5 min of age.

- Breastfeeding rates.

- Macrosomia (birth weight $\geq 4000 \mathrm{~g}$ )

- Large for gestational age (birth weight $\geq 90$ th centile).

- Admission to neonatal intensive care unit (NICU) or special care nursery (SCN).

- Congenital anomalies.

Statewide billing for relevant Medicare Benefits Schedule item numbers

The quarterly number of antenatal and postnatal consultations performed by a doctor, nurse, midwife or an Aboriginal and Torres Strait Islander health practitioner will be estimated from Medicare Benefits Schedule (MBS) statistics available at the Australian Department of Health website (http://medicarestatistics.humanservices.gov.au/statistics/mbs_item.jsp). We will also collect MBS item numbers for COVID-19 Temporary MBS Telehealth services and obstetric ultrasound billing. See online supplemental appendicies 3 and 4 for details.
DATA COLLECTION PROCEDURES

\section{Description of procedures}

1. During the third week of each month, perinatal outcomes for the preceding calendar month will be extracted from the BOS (or equivalent) by site investigators at each health service. The data will be deidentified and uploaded to a secure online REDCap $^{\mathrm{TM}}$ database administered by the University of Melbourne.

2. MBS item numbers for Victoria by quarter will be downloaded from the Australian Department of Health website and collated by the central study team. http://medicarestatistics.humanservices.gov.au/statistics/mbs_item.jsp.

3. Once complete, the report will be disseminated to all investigators by email.

The collaborative maternity and newborn dashboard (CoMaND) project is designed to be adaptive to rapid changes in the healthcare environment. Therefore, the reported indicators may be adjusted during the course of the study in order to respond to potential areas of concern (eg, emerging high-risk groups that require specific monitoring).

\section{Data collection and management}

The coordinating principal investigator (CPI) will be responsible for storing essential study documents relevant to central data management and maintaining sitespecific data submission records. A data collection log and instruction manual will be maintained to record all handling of submitted data from each site. The central study team will merge and analyse the centralised data for the report.

Study data will be collected and managed using REDCap electronic data capture tools ${ }^{12}$ hosted at The University of Melbourne. Data are backed up nightly to a local backup server. REDCap maintains an audit trail of data create/update/delete events that is accessible to project users who are granted permission to view it. Access to REDCap will be provided via The University of Melbourne user account or (for external collaborators) via a REDCap user account created by the University of Melbourne system administrator. Working files for central data clean up, merging, analysis and storage will be held on password-protected computers and archived on University of Melbourne servers.

\section{Statistical methods}

Statistical analyses will be performed on the data collected from the CoMaND reports in two steps: (1) Analyses for routine clinical quality reporting and (2) Statistical analyses for research purposes.

\section{Analyses for clinical quality reporting}

The primary aim of this data collection is to detect any significant deviations in performance indicators over time, relative to the baseline prepandemic medians. The prepandemic medians will be calculated from the period encompassing 1 January 2018 to 31 March 2020 (onset 
of stage 3 lockdown). Deidentified individual patientlevel data on $>100$ fields will be analysed in STATA SE V.16. ${ }^{13}$ Weekly outcomes will be displayed as run charts according to established methods for detecting nonrandom 'signals' in healthcare as described elsewhere. ${ }^{14}$ Interpretations will be made according to probabilitybased rules for non-random patterns of data (alpha error of $<0.05$ ). The signal will be categorised as 'shift' if there are $\geq 6$ consecutive weeks all above or below the prepandemic median. A 'trend' is defined as $\geq 5$ consecutive weeks continuously going all up or all down. Detecting a signal suggesting deviation from a stable baseline will prompt communication to participating health services to allow further scrutiny for possibly contributory factors and areas for improvement.

\section{Statistical analyses for research purposes}

Further in-depth analyses will be performed (ie, survival analysis for preterm births, multivariate logistic regression analyses, time-series analyses for all other outcomes etc) to determine the effects of lockdowns on the outcomes of interests. We will compare the maternal, perinatal and neonatal outcomes between the cohort of women who were pregnant during the pandemic restrictions in metropolitan Melbourne with those that gave birth before the onset of restrictions. We will define lockdown exposure period as those weeks where national stringency index was $\geq 50$ on the Oxford COVID-19 Government Response Tracker scale for at least 5 days per week. ${ }^{15}$ The Oxford COVID-19 Government Response Tracker provides a systematic cross-national, cross-temporal measure to document government responses during the pandemic. Melbourne had the biggest COVID-19 caseload nationally throughout 2020 and therefore drove the Australian government stringency index in that year. The national stringency index threshold of 50 was used in accordance with the definition of lockdown in the International Perinatal Outcomes in the Pandemic study. ${ }^{15}$

The outcomes to be analysed include preterm births (iatrogenic and spontaneous), stillbirths, Apgars <5, admission to NICU or SCN, fetal growth restriction and macrosomia.

All pregnancies resulting to a singleton birth of at least 20 weeks gestation will be included whereas those with major anomalies and terminated pregnancies will be excluded.

Various covariates will also be used to adjust the multivariate analyses performed on each maternal and perinatal outcome. These covariates include: maternal age $(<25,25-29,30-34,35-39,40+$ years $)$, region of birth (Australian Bureau of Statistics classifications), interpreter required (yes, no), parity $(0,1,2, \geq 3)$, maternal body mass index, Socio-Economic Indexes For Areas (SEIFA 2016): Index of Relative Socioeconomic Advantage and Disadvantage scores for the maternal postcode of residence, smoking status.
ETHICS AND DISSEMINATION

\section{Ethics approvals}

Ethics approvals for the CoMaND project have been obtained from the Austin Health (HREC/64722/Austin2020) and Mercy Health (ref. 2020-031). As this is a retrospective review of de-identified health information, there was no requirement for individual consent, or a waiver of consent.

\section{Dissemination}

Clinical quality indicator reports: 'CoMaND reports'

Periodic reports containing the maternal and fetal/ neonatal outcomes listed in the methods will be provided to the following people listed below each month or as otherwise deemed practicable.

- CPI and site PIs.

- Chair of the Melbourne Academic Centres for Health, Women's and Newborn network.

- Senior maternity advisor to SCV/Chair of Consultative Council of Obstetric and Paediatric Morbidity and Mortality, who will disseminate to the members of the COVID-19 expert obstetric advisory group to SCV.

Authorised representatives of the sponsoring institution as well as representatives from the human research ethics committees, research governance office and regulatory agencies may inspect all documents and records required to be maintained by the CPI in this study.

\section{Research publications}

In addition to the regular report circulation as described above, results may also be written up for journal publication and/or presentation at scientific conferences. Wherever possible, research publications will be published under an open access policy. Data sharing with international investigators will be in accordance with relevant ethics approvals. The results will be distributed to the relevant clinical service leaders, who will translate the findings into clinical guidelines and protocols as appropriate.

\section{Data confidentiality}

No individually identifying data will be collected or stored. Outcomes will be reported in aggregate forms, and results will not be disseminated in a format that allows the identification of an individual patient.

\section{Archiving: data and document retention}

Data will be stored indefinitely. The CPI will be the custodian of the data. Local site study documents will be stored for at least 15 years and will not be destroyed without the written consent of the CPI. The CPI will inform Principal Site Investigators when these documents no longer need to be retained. Secure destruction of research documents using software to permanently erase the data will be utilised at the end of the archive period.

\section{Data sharing}

National and international collaborators

The study investigators may contribute aggregate and de-identified individual patient data to national and 
international collaborations whose proposed use of the data has been ethically reviewed and approved by an independent committee and following the signing of an appropriate research collaboration agreement.

\section{Data availability statements for research publications}

Following the ethical obligations for data sharing outlined by the International Committee for Medical Journal Editors, ${ }^{16}$ the following data sharing arrangements will be permitted:

After article publication, the following study data will be made available to researchers from a recognised research institution whose proposed use of the data has been ethically reviewed and approved by an independent committee.

- Individual participant data that underlie the results reported in the article, after deidentification (text, tables, figures and appendices).

- The study protocol, statistical analysis plan, analytical code.

Proposals should be directed to the chief investigator at lisa.hui@unimelb.edu.au. To gain access, data requestors will need to sign a data access agreement with the University of Melbourne.

\section{Patient and public involvement}

No patient was involved in the design of this study. The development of the outcome measures was informed by the Victorian Department of Health and Human Services PSPI. ${ }^{8}$

\section{DISCUSSION}

This project is the first clinician-led, multicentre maternity data collection system for metropolitan Melbourne. It complements the statewide government maternity data, with the major benefits of more timely and flexible reporting of outcomes and granular detail on emerging areas of concern. This resource will support datainformed hospital pandemic or postpandemic responses through to the end of 2022.

Overall, the quality of the Victorian perinatal data collection is considered high. ${ }^{17}$ However, there are limitations to the data collection methods, including missing data on planned home births, private hospital births and COVID-19 infection rates in pregnancy. There are also hospital-specific variations to the many data collection fields, requiring manual recoding and collation before indicators can be generated.

There are potential risks related to data security. However, as no personally identifying information will be collected in this study, there is no possibility of breaches of privacy or confidentiality. The risks to the successful conduct of the study include inadequate health service resources to collect and submit the data or insufficient study resources to clean and analyse data on a continuous basis. We have designed this study to leverage existing routine data reporting tools to minimise the burden on health services. We have also obtained philanthropic and University funding to support the central data management and report generation (see Funding declarations).

\section{SIGNIFICANCE}

This collaboration will create a clinician-led resource for data-informed health policy in maternity care. The statistical power of this data collection for rare outcomes such as stillbirth is enhanced by its large sample size and the complete public hospital coverage of the Australian city most affected by pandemic restrictions in 2020. It seamlessly complements the existing department of health state-wide data collection without creating redundancies and onerous workloads for hospital data managers. The system benefits from the advantages of being more agile than existing data sources. The active monthly data collection also allows rapid adjustment of reported indicators to address clinical concerns as they emerge. It is anticipated that this resource will be of enduring value to clinical leaders in maternity health services beyond the peak of the COVID-19 pandemic. We propose patient and public involvement in the translation of our research findings to ensure that the perspectives of women and other stakeholders are incorporated into any future recommendations for clinical care.

\section{Author affiliations}

${ }^{1}$ Department of Obstetrics and Gynaecology, Melbourne Medical School, The University of Melbourne, Melbourne, Victoria, Australia

${ }^{2}$ Department of Perinatal Medicine, Mercy Hospital for Women, Heidelberg, Victoria, Australia

${ }^{3}$ Department of Obstetrics and Gynaecology, The Northern Hospital, Northern Health, Epping, Victoria, Australia

${ }^{4}$ Reproductive Epidemiology Group, Murdoch Children's Research Institute, Parkville, Victoria, Australia

${ }^{5}$ Department of Obstetrics and Gynaecology, Mercy Hospital for Women, Heidelberg, Victoria, Australia

${ }^{6}$ Department of Obstetrics and Gynaecology, Monash University, Clayton, Victoria, Australia

${ }^{7}$ Department of Obstetrics and Gynaecology, Monash Medical Centre, Monash Health, Clayton, Victoria, Australia

${ }^{8}$ Department of Maternal-Fetal Medicine, Joan Kirner Women's and Children's Hospital, Western Health, St Albans, Victoria, Australia

${ }^{9}$ Department of Obstetrics and Gynaecology, The Royal Women's Hospital, Parkville, Victoria, Australia

${ }^{10}$ Department of Obstetrics and Gynaecology, Box Hill Hospital, Eastern Health, Box Hill, Victoria, Australia

${ }^{11}$ Department of Obstetrics and Gynaecology, Frankston Hospital, Peninsula Health, Frankston, Victoria, Australia

\section{Twitter Lisa Hui @LisaHui4}

Acknowledgements We also acknowledge the contribution of the following hospital staff for their assistance in setting up the data collection within their respective health services: Tania Fletcher (Mercy Health), Michelle Knight (Monash Health), Lynne Rigg (Royal Women's Hospital), Abby Monaghan (Northern Health), Lee-Anne Lynch (Western Health), Pauline Hamilton (Eastern Health) and Roshanee Perera (Peninsula Health).

Contributors LH, SPW, JMS, CLW, DLR, BWM, KRP and SP conceived of the original study protocol, LH designed, coordinated and acquired funding for the study; LH, MBM, SPW, JMS, CLW, DLR, BWM, KRP, JF, PMS, NP and SP contributed to the data analysis plan; LH and SP obtained ethics approvals; KRP, CLW, JMS, JF, PMS, LH and SP obtained site specific approvals; LH wrote the primary manuscript; 
all authors reviewed and edited the draft manuscript for intellectual content and approved the final submitted manuscript.

Funding This project is funded by a philanthropic grant from the Norman Beischer Medical Research Foundation (Grant No. N/A) and a University of Melbourne Department of O\&G Innovation grant to LH (Grant No. N/A).

Disclaimer The investigators have no financial or competing interests for the overall study.

Competing interests BWM reports consultancy for ObsEva and received research funding from Ferring and Merck. KRP has received consultancy fees from GSK and Janssen Pharmaceuticals. LH has received research funding from Ferring Pharmaceuticals outside the scope of this study. All other investigators do not declare any financial or competing interests for the overall study.

Patient consent for publication Not applicable.

Provenance and peer review Not commissioned; externally peer reviewed.

Supplemental material This content has been supplied by the author(s). It has not been vetted by BMJ Publishing Group Limited (BMJ) and may not have been peer-reviewed. Any opinions or recommendations discussed are solely those of the author(s) and are not endorsed by BMJ. BMJ disclaims all liability and responsibility arising from any reliance placed on the content. Where the content includes any translated material, BMJ does not warrant the accuracy and reliability of the translations (including but not limited to local regulations, clinical guidelines, terminology, drug names and drug dosages), and is not responsible for any error and/or omissions arising from translation and adaptation or otherwise.

Open access This is an open access article distributed in accordance with the Creative Commons Attribution Non Commercial (CC BY-NC 4.0) license, which permits others to distribute, remix, adapt, build upon this work non-commercially, and license their derivative works on different terms, provided the original work is properly cited, appropriate credit is given, any changes made indicated, and the use is non-commercial. See: http://creativecommons.org/licenses/by-nc/4.0/.

\section{ORCID iDs}

Lisa Hui http://orcid.org/0000-0002-9720-3562

Daniel L Rolnik http://orcid.org/0000-0002-2263-3592

Joanne M Said http://orcid.org/0000-0001-6263-0030

Kirsten R Palmer http://orcid.org/0000-0001-7421-6291

Ben W Mol http://orcid.org/0000-0001-8337-550X

Susan P Walker http://orcid.org/0000-0001-9075-4655

\section{REFERENCES}

1 Hedermann G, Hedley PL, Bækvad-Hansen M, et al. Danish premature birth rates during the COVID-19 lockdown. Arch Dis Child Fetal Neonatal Ed 2021;106:93-5.
2 Matheson A, McGannon CJ, Malhotra A, et al. Prematurity rates during the coronavirus disease 2019 (COVID-19) pandemic lockdown in Melbourne, Australia. Obstet Gynecol 2021;137:405-7.

3 Philip RK, Purtill H, Reidy E, et al. Unprecedented reduction in births of very low birthweight (VLBW) and extremely low birthweight (ELBW) infants during the COVID-19 lockdown in Ireland: a 'natural experiment' allowing analysis of data from the prior two decades. BMJ Glob Health 2020;5:e003075.

4 Roberton T, Carter ED, Chou VB, et al. Early estimates of the indirect effects of the COVID-19 pandemic on maternal and child mortality in low-income and middle-income countries: a modelling study. Lancet Glob Health 2020;8:e901-8.

5 Hale T. Oxford COVID-19 Government response rracker. Blavatnik School of Government. Data use policy: Creative Commons Attribution CC BY standard, 2020.

6 Victoria SC. Consultative Council on obstetrics and paediatric mortality and morbidity. In: Victoria's mothers, babies and children in 2019. 2019, 2021

7 The Royal Australian and New Zealand College of obstetricians and gynaecologists. advice to obstetricians and gynaecologists, GP obstetricians, 2020. Available: https://ranzcog.edu.au/news/adviceto-obstetricians-and-gynaecologists,-gp-obs [Accessed 8 Mar 2021].

8 Hunt R. Victorian perinatal services performance indicators 2018-19. Safer Care Victoria: Melbourne, Victoria, 2019.

9 World Health Organization,. COVID 19 public health emergency of international concern (PHEIC). global research and innovation Forum: towards a research roadmap, 2020.

10 Department of Health and Human Services. Capability frameworks for Victorian maternity and newborn services. Melbourne, Victoria, Australia: Department of Health and Human Services, DHHS, 2019.

11 Australian Institute of Health and Welfare. Women who gave birth, by maternal age group and state and territory of birth, 2018, 2018. Available: https://www.aihw.gov.au/reports/mothersbabies/australias-mothers-babies-data-visualisations/contents/ demographics-of-mothers-and-babies/maternal-age [Accessed 5 Mar 2021].

12 Harris PA, Taylor R, Thielke R, et al. Research electronic data capture (REDCap) - a metadata-driven methodology and workflow process for providing translational research informatics support. J Biomed Inform 2009;42:377-81.

13 StataCorp. Stata 16 SE. College Station, Texas: StataCorp, 2019.

14 Perla RJ, Provost LP, Murray SK. The run chart: a simple analytical tool for learning from variation in healthcare processes. BMJ Qual Saf 2011;20:46-51.

15 Stock SJ, Zoega H, Brockway M, et al. The International perinatal outcomes in the pandemic (iPOP) study: protocol. Wellcome Open Res 2021;6:21.

16 Taichman DB, Sahni P, Pinborg A, et al. Data sharing statements for clinical trials: a requirement of the International Committee of medical journal editors. Ann Intern Med 2017;167:63-5.

17 Flood MM, McDonald SJ, Pollock WE, et al. Data accuracy in the Victorian perinatal data collection: results of a validation study of 2011 data. Health Inf Manag 2017;46:113-26. 